01,19

\title{
О связи свойств металлических стекол и материнских кристаллов
}

\author{
() Ю.П. Митрофранов ${ }^{1}$, Н.П. Кобелев ${ }^{2}$, В.А. Хоник ${ }^{1}$ \\ ${ }^{1}$ Воронежский государственный педагогический университет, \\ Воронеж, Россия \\ ${ }^{2}$ Институт фризики твердого тела РАН, \\ Черноголовка, Россия \\ E-mail: mitrofanovyup@gmail.com
}

Поступила в Редакцию 24 января 2019 г.

В окончательной редакции 24 января 2019 г.

Принята к публикации 5 фревраля 2019 г.

\begin{abstract}
Можно обоснованно ожидать, что свойства металлических стекол должны быть связаны со свойствами материнских кристаллов, использованных для их изготовления. Этот вопрос, однако, в научной литературе не поднимался, за единичными исключениями. В настоящей работе кратко суммированы результаты сопоставления изменений модуля сдвига, плотности и кинетики тепловых эффектов в металлических стеклах при структурной релаксации, в состоянии переохлажденной жидкости и при кристаллизации. Продемонстрировано, что величины тепловых эффектов, как и изменения плотности, могут быть определены исходя из изменений модуля сдвига стекла по отношению к модулю сдвига материнского кристалла, что свидетельствует о генетической связи свойств стекла со свойствами материнского кристалла. При этом материнский кристалл в энергетическом смысле можно интерпретировать как основное состояние стекла, избыточная внутренняя энергия которого определяется упругой энергией системы ,дефектов“ типа межузельных гантелей, возникающих при плавлении и последующей закалке расплава.
\end{abstract}

Работа поддержана грантом Минобрнауки РФ № 3.1310.2017/4.6.

DOI: 10.21883/FTT.2019.06.47676.365

\section{1. Введение}

Металлические стекла (МС) являются объектом неослабевающего интереса $[1,2]$. Это связано как с чисто научными аспектами, так и с расширением областей технического применения МС. Усилия исследователей в последние годы привели к накоплению многосторонней информации о структуре и физических свойствах этих материалов, что нашло отражение в ряде недавних обзоров [3-6]. Основное внимание при этом уделяется определению особенностей структурного состояния и выявлению связи „структура $\rightarrow$ свойства“. Некристалличность структуры определяет избыточную энергию Гиббса и, как следствие, реализацию многоплановых релаксационных процессов, значительно изменяющих как саму структуру, так и физические свойства МС. Стандартный подход к интерпретации этих релаксационных процессов состоит в выделении некоторой системы локализованных „дефектов“, термическая (или термомеханическая) активация которых с переходом в новое структурное состояние определяет макроскопическую релаксацию структуры и свойств.

Наиболее популярный подход связывает „дефекты“ (кавычки для простоты далее опущены) с областями избыточного свободного объема (free volume), которые обычно понимаются просто как области пониженной плотности [7,8]. Предполагается, что изменения избыточного свободного объема вызывают релаксацию различных физических свойств МС при термообра- ботке. Свободный объем, однако, не имеет однозначного количественного определения и основанные на нем представления подвергались неоднократной критике $[9,10]$. Модель зон сдвиговых превращений (shear transformation zones) предполагает перестройку групп атомов, сопровождающуюся локальной дилатацией [11]. Похожий подход реализован в модели „мягких зон“ (soft zones) [12]. Некоторые общие черты этих подходов сочетаются в модели „единиц течения“ (flow units), согласно которой дефекты структуры рассматриваются как жидкоподобные области пониженной плотности с уменьшенным локальным модулем сдвига [13].

Указанные представления (а также ряд других) в той или степени успешно применялись для интерпретации различных особенностей структурной релаксации и пластической деформации МС. Общим аспектом этих представлений является в целом искусственный подход к определению дефектов, реализуемый наиболее часто по результатам компьютерного моделирования (молекулярная динамика/статика) или построения физических моделей (например, систем коллоидных или других частиц, моделирующих межатомное взаимодействие). В силу этого представления о дефектах в МC не обладают наглядностью и убедительностью в той степени, как это имеет место в случае кристаллов. Кроме того, если дефекты в кристаллах органически связаны с кристаллической решеткой, то имеющиеся представления о дефектах в МС какой-либо связи с материнским кристаллом (или с каким-либо другим состоянием, являющемся 
FCC

100 dumbbell

$(\mathrm{Al}, \mathrm{Cu}, \mathrm{Ni})$

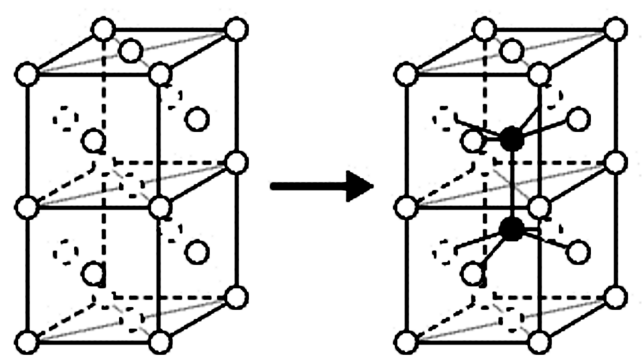

$\mathrm{BCC}$

110 dumbbell

$\mathrm{Fe}$

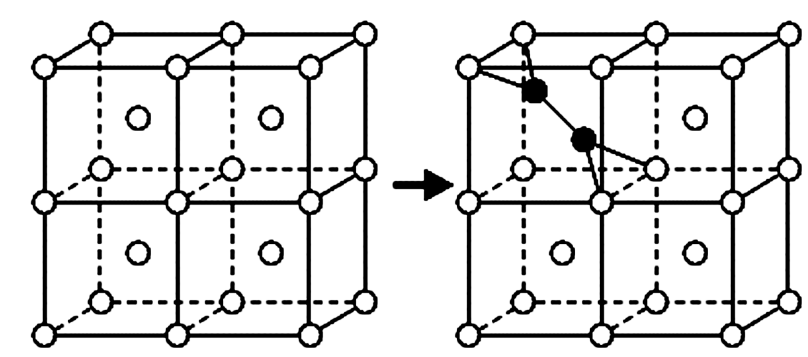

$\mathrm{HCP}$

001 dumbbell $=0001$ dumbbell

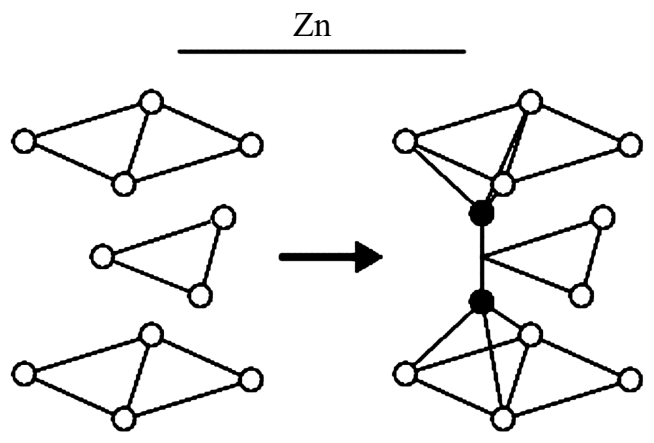

Рис. 1. Межузельные гантели в различных кристаллических структурах [18].

основным в энергетическом смысле) не предполагают, за единственным исключением, рассмотренным ниже. Между тем вполне очевидно, что такая связь должна существовать. Действительно, например, свойства МС системы $\mathrm{PdNiCuP}$ и их релаксация при термообработке должны быть с необходимостью связанными со свойствами интерметаллических и/или фосфидных кристаллических фаз в этой системе. Насколько нам известно, вопрос о наличии такой связи в рамках вышеупомянутых и других моделей даже не поднимался. Принципиальная задача поэтому состоит в том, чтобы наличие этой связи выяснить.

В 1992 г. Э. Гранато на основе результатов многолетних исследований дефектов в простых металлических кристаллах и термодинамических расчетов предложил межузельную теорию (МТ) [14]. Изначально предполагалось, что МТ объединит кристаллическое, жидкое (равновесное и переохлажденное) и некристаллическое твердое (стеклообразное) состояния. Авторы настоящей работы в течение длительного времени использовали МТ в качестве идеологической основы для интерпретации различных релаксационных явлений в МС. При этом, в частности, было показано, что описание релаксации свойств МС на основе МТ включает свойства материнского кристалла естественным и неотъемлемым образом. Этот вывод, однако, ранее не акцентировался, хотя его значимость заслуживает специального обобщения. Цель настоящей работы состоит в суммировании некоторых результатов, свидетельствующих о наличии связи свойств МС и материнских кристаллов. Сначала будут кратко изложены основы МТ, а затем приведены результаты экспериментов и их интерпретация, дающих аргументы в пользу указанного вывода.

\section{2. Основы межузельной теории}

Исследования влияния мягкого нейтронного облучения на упругие свойства монокристаллов меди, выполненные в 70-х годах прошлого века, привели Гранато с сотрудниками и ряда других авторов к выводу о том, что межузельные атомы в кристаллической решетке образуются в так называемой гантельной конфигурации, когда два атома стремятся занять один и тот же узел кристаллической решетки $[15,16]$. В настоящее время считается установленным, что межузельные атомы в различных кристаллических решетках действительно образуют гантельные конфигурации (межузельные гантели), являющиеся наиболее энергетически выгодными [17]. Примеры межузельных гантелей представлены на рис. 1 [18]. Гранато также показал, что межузельные гантели вызывают довольно сильное снижение нерелаксированного („мгновенного“) модуля сдвига: эффект, 
получивший название „диаэластический“, примерно на порядок большее, чем для вакансии [14]. Экстраполяция обнаруженного диаэластического эффекта в область высоких концентраций показала, что если окажется возможным каким-то способом создать концентрацию межузельных гантелей в $2-3 \%$, то модуль сдвига металла будет стремиться к нулю [14]. Нулевой (или близкий к нулю) модуль сдвига характерен для жидкости [19]. Это привело Гранато к предположению о том, что плавление металлов связано с генерацией межузельных атомов. Специально поставленные недавние эксперименты на $\mathrm{Al}$ и In подтвердили это предположение [20,21]. Компьютерное моделирование показало, что межузельные гантели остаются идентифицируемыми структурными объектами как в жидком [22], так и в твердом некристаллическом состоянии, полученном закалкой расплава [23,24].

Гранато вычислил энергию Гиббса исходного кристаллического материала в зависимости от концентрации межузельных гантелей и показал, что при относительно низких гомологических температурах термодинамически устойчиво состояние с малой концентрацией гантелей, тогда как выше температуры плавления устойчиво состояние с их концентрацией в несколько процентов $[14,25]$. Первое состояние было интерпретировано как равновесный кристалл с дефектами, второе - как равновесная жидкость. При этом оказались термодинамически возможны метастабильные состояния перегретого кристалла и переохлажденной жидкости. Соответственно, твердое некристаллическое состояние (то есть металлическое стекло) интерпретировалось как замороженная жидкость, содержащая межузельные дефекты [26]. Тогда релаксационные явления в стекле в рамках этого подхода должны быть связаны с изменением концентрации или энергии этих дефектов.

Межузельные гантели в кристаллических металлах обладают следующими основными свойствами. Во-первых, это высокая чувствительность к внешнему сдвиговому напряжению, которое вызывает струноподобное движение около двух десятков атомов вокруг ядра дефекта, приводя к значительному неупругому снижению модуля сдвига [14,27]. Во-вторых, колебательная плотность состояний атомов дефекта содержит ярко выраженные низкочастотные (ниже дебаевской частоты $\omega_{D}$ ) и высокочастотные (выше $\omega_{D}$ ) колебательные моды $[15,28]$. С низкочастотными колебательными модами связана высокая энтропия образования дефекта, что позволяет объяснить правило плавления Ричардса энтропия плавления равна $\approx 1.2 k_{B}\left(k_{B}-\right.$ постоянная Больцмана) в расчете на один атом для всех элементов периодической системы, за небольшими исключениями [29]. Наконец, в-третьих, межузельные гантели создают специфические сдвиговые поля напряжений [14].

Принципиальный вопрос состоит в подтверждении наличия межузельных дефектов, или им подобных, в жидком и стеклообразном состояниях. Помимо упомянутой работы [22], специально поставленной для идентификации межузельных гантелей в жидкости, в много- численных работах по компьютерному моделированию жидкостей многократно отмечалось наличие „динамических гетерогенностей“, „кооперативно перестраивающихся областей“ и других структурных особенностей, которые сходны с межузельными гантелями в кристаллах [30,31]. Компьютерное моделирование стеклообразной меди и алюминия показало, что в моделях действительно присутствуют области, свойства которых близки к свойствам межузельных гантелей в кристаллах $[23,32]$. В отличие от кристалла, однако, они не имеют характерного геометрического образа. Таким образом, можно предполагать, что дефекты, аналогичные межузельным гантелям в кристаллах, существуют и в жидком, и в твердом некристаллических состояниях. В дальнейшем, имея в виду изложенное, мы будем называть их для простоты межузельными гантелями.

Принимая изложенный концептуальный подход, можно сформулировать аналитические выражения, определяющие свойства МС в рамках МТ. По существу, это всего лишь два простых уравнения. Первое из них отражает вышеупомянутый диаэластический эффект снижение нерелаксированного модуля сдвига с ростом концентрации межузельных гантелей [14,25]:

$$
G=\mu \exp (-\alpha \beta c),
$$

где безразмерный параметр $\alpha \approx 1$ связан с полем деформации дефекта [33], безразмерная сдвиговая восприимчивость $\beta$ (обычно равная 18-20 для различных МC) характеризует чувствительность модуля сдвига к концентрации межузельных гантелей и связана с ангармонизмом потенциала межатомного взаимодействия [34], $\mu$ - модуль сдвига материнского кристалла (то есть кристалла, используемого для приготовления МС закалкой расплава). При этом неважно, является ли этот кристалл однофазной или многофазной системой. Поскольку при нулевой концентрации дефектов $G=\mu$, то материнский кристалл является основным (бездефектным) состоянием стекла. В контексте настоящей работы это утверждение является принципиально важным и, по существу, определяет связь свойств стекла и материнского кристалла.

Второе базовое уравнение МТ говорит о том, что энтальпия $H$ образования межузельного дефекта пропорциональна модулю сдвига $G$ [14,25]:

$$
H=\alpha \Omega G,
$$

где $\alpha$ - та же величина, что и в формуле (1), а $\Omega$ - объем в расчете на атом. Формулы (1) и (2) имеют далеко идущие следствия. Во-первых, согласно уравнению (1), энтальпия формирования дефекта (2) также связана с материнским кристаллом. Во-вторых, если в результате релаксационных процессов меняется концентрация дефектов $c$, то при этом поглощается или выделяется (в зависимости от знака изменения $c$ ) их суммарная энтальпия формирования. Это прямо означает, что любые релаксационные явления в МС должны сопровождаться тепловыми эффектами. К этому 
же эффекту приводит и изменение величины $\alpha$ в формулах (1) и (2), которое может быть связано с изменением локальной структуры дефекта, вызывающего изменение поля упругой деформации вокруг него. Можно показать, что величину $\alpha c$ можно рассматривать как эффективную концентрацию дефектов.

Применение изложенного подхода к жидкостям (как равновесным, так и переохлажденным) и к МС дает правильную интерпретацию целого ряда релаксационных явлений. Соответствующие обзоры приведены в работах $[29,35]$. Мы ограничимся кратким описанием наиболее ярких результатов, полученных при интерпретации релаксационных явлений в МС в рамках МТ, которые демонстрируют связь свойств МС и материнских кристаллов. Прежде всего, это касается связи релаксации дефектной структуры с тепловыми явлениями в МС.

\section{3. Кинетика тепловых эффектов при структурной релаксации, в состоянии переохлажденной жидкости и при кристаллизации}

Согласно уравнению (1), кинетика изменений концентрации дефектов в результате релаксационных явлений может быть отслежена посредством прецизионных измерений модуля сдвига. Как отмечено выше, любое изменение этой концентрации (или величины $\alpha c$ ) должно вызывать тепловые эффекты, в соответствии с уравнением (2). Совместное использование этих уравнений приводит к выражению для теплового потока $W$ (измеряемого в $\mathrm{W} / \mathrm{kg}$ ) в процессе линейного нагрева МC с постоянной скоростью $\dot{T}$ [33]:

$$
W=\frac{\dot{T}}{\beta \rho}\left[\frac{G}{\mu} \frac{d \mu}{d T}-\frac{d G}{d T}\right],
$$

где $\rho$ - плотность, смысл остальных величин указан выше. Поскольку плотность с точностью $\approx 1 \%$ остается постоянной, скорость нагрева задана, а сдвиговую восприимчивость можно определить из анализа экспериментальных данных [34], то для расчета теплового потока необходимо знать модули сдвига стекла и материнского кристалла в зависимости от температуры. Подчеркнем, что величина $W$, описываемая формулой (3), непосредственно измеряется в эксперименте методом дифференциальной сканирующей калориметрии (ДСК). Качество измерения зависимостей $G(T)$ и $\mu(T)$ для расчета теплового потока по формуле (3) должно быть весьма высоким, поскольку это соотношение включает как производные модулей сдвига $G$ и $\mu$ по температуре, так и разность этих производных. Пример таких высокоточных измерений модуля сдвига МС в исходном состоянии и после полной кристаллизации показан на рис. 2, который демонстрирует характерное поведение $G$ [36]. Нагрев исходного стекла первоначально вызывает чисто ангармоническое снижение модуля сдвига $G$, которое далее замедляется вследствие интенсивной структурной релаксации, вызывающей рост $G$. Вблизи калориметрической температуры стеклования $T_{g}$ начинается интенсивное падение модуля сдвига, отражающее переход вещества в состояние переохлажденной жидкости. При дальнейшем нагреве при некоторой температуре $T_{x}>T_{g}$ начинается многостадийная кристаллизация, вызывающая необратимый рост $G$. После полной кристаллизации имеет место линейное ангармоническое снижение модуля сдвига с температурой [36].

Показанные на рис. 2 зависимости $G(T)$ и $\mu(T)$ могут быть непосредственно использованы для расчета теплового потока по формуле (3). Результат расчета показан на рис. 3 сплошной кривой. Штриховой кривой

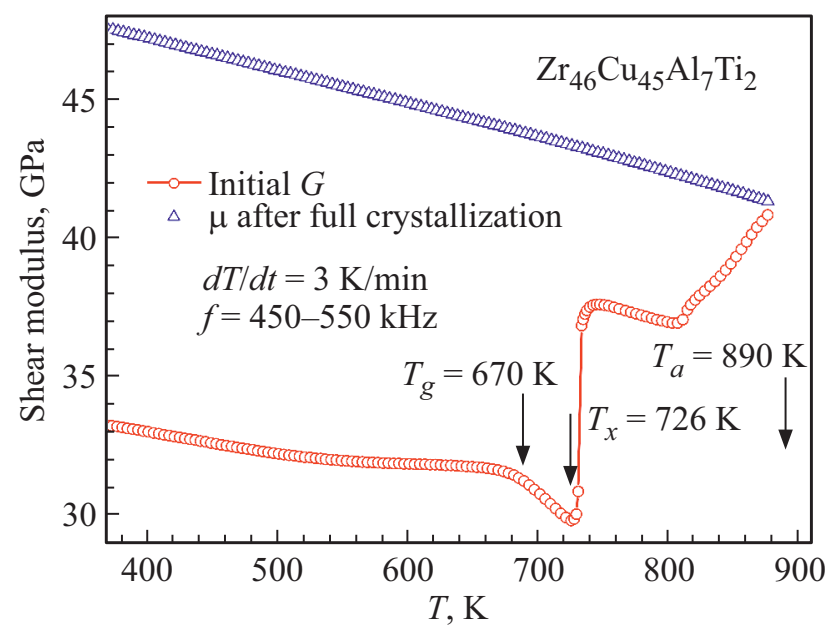

Рис. 2. Температурные зависимости модуля сдвига МС $\mathrm{Zr}_{46} \mathrm{Cu}_{45} \mathrm{Al}_{7} \mathrm{Ti}_{2}$ в исходном состоянии и после полной кристаллизации при $T_{a}=890 \mathrm{~K}$, измеренные при скорости нагрева $3 \mathrm{~K} / \mathrm{min}$ [36]. Стрелками показаны температуры стеклования $T_{g}$, начала кристаллизации $T_{x}$ и кристаллизационного отжига $T_{a}$.

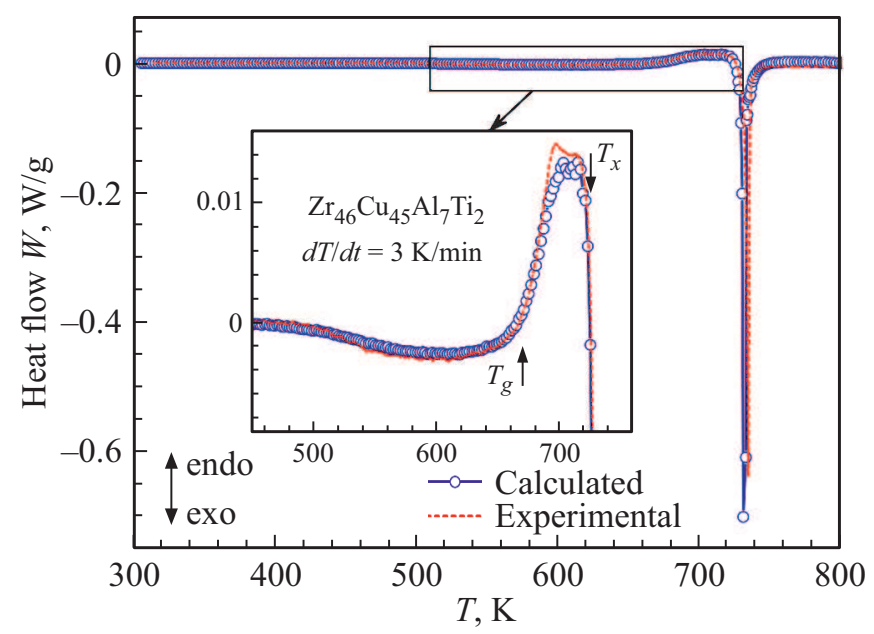

Рис. 3. Экспериментальная термограмма ДСК, измеренная при нагреве $\mathrm{MC} \mathrm{Zr}_{46} \mathrm{Cu}_{45} \mathrm{Al}_{7} \mathrm{Ti}_{2}$ со скоростью $3 \mathrm{~K} / \mathrm{min}$, совместно с тепловым потоком, рассчитанным по формуле (3) на основе экспериментальных температурных зависимостей модуля сдвига, показанных на рис. 2 [36]. 
на этом рисунке представлена экспериментальная кривая ДСК того же стекла, не подвергавшаяся какой-либо обработке, за исключением обычной коррекции базовой линии калориметра. На вставке показаны расчетные и экспериментальные кривые в увеличенном масштабе. Как видно, имеет место отличное соответствие расчета эксперименту [36]. Расчет хорошо воспроизводит экзотермическую тепловую реакцию ниже температуру стеклования $T_{g}$, эндотермическую реакцию в состоянии переохлажденной жидкости (то есть при $T_{g} \leq T \leq T_{x}$ ), а также экзотермическую реакцию при кристаллизации.

Из представленных на рис. 3 результатов можно сделать следующие выводы. Во-первых, формула (3) практически адекватно описывает эксперимент, но при этом по-существу не содержит каких-либо подгоночных параметров. Во-вторых, действительно, тепловой поток при нагреве МС контролируется как модулем сдвига стекла, так и модулем сдвига материнского кристалла. Подстановка в формулу (3) зависимостей $G(T)$ и $\mu(T)$, отличных от показанных на рис. 2, не дает скольконибудь удовлетворительного описания эксперимента. Наконец, в-третьих, довольно неожиданным оказался факт применимости уравнения (3) не только собственно для аморфного состояния (то есть ниже температуры $\left.T_{x}\right)$, но и для частичной кристаллизации. Этот результат предполагает, что экзотермический тепловой эффект кристаллизации обусловлен падением концентрации дефектов до нуля. Другими словами, природа теплоты кристаллизации может быть связана с переходом упругой энергии системы дефектов в тепло. Это предположение можно проверить в специальном эксперименте.

\section{4. Тепловыделение при кристаллизации металлического стекла}

Пренебрегая изменением плотности при кристаллизации, разность внутренних энергий на единицу объема МС и материнского кристалла может быть вычислена в рамках МТ как $[33,37]$

$$
\rho \Delta U=\frac{\mu}{\beta}\left[1-\frac{G}{\mu}\right],
$$

где все входящие в эту формулу величины были определены выше. Как видно, избыточная внутренняя энергия МС по отношению к материнскому кристаллу определяется просто соответствующими модулями сдвига $G$ и $\mu$. По своему физическому смыслу величина $\rho \Delta U$ в формуле (4) есть упругая энергия системы дефектов межузельного типа [33]. Если, как отмечено выше, теплота кристаллизации определяется переходом этой упругой энергии в тепло, то величина $\rho \Delta U$ должна быть равна теплоте кристаллизации $\rho Q_{x}$. Калориметрические измерения теплот кристаллизации ряда МС совместно с измерениями скачка модуля сдвига при кристаллизации

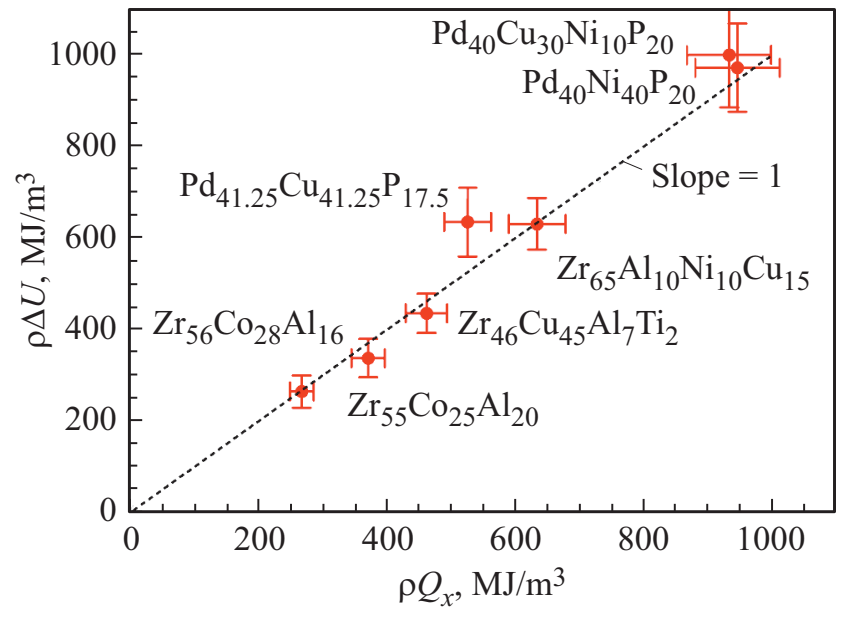

Рис. 4. Вызванное кристаллизацией изменение внутренней энергии на единицу объема $\rho \Delta U$, вычисленное по формуле (4), в зависимости от теплоты кристаллизации на единицу объема $\rho Q_{c r}$ для указанных МС [37]. Штриховая линия соответствует единичному наклону.

были представлены в работе [37]. Результаты этих измерений в виде зависимости $\rho \Delta J$ от $\rho Q_{x}$ представлены на рис. 4. Прямая линия на этом рисунке проведена с единичным наклоном. Из него следует, что, с учетом погрешностей измерений, действительно $\rho \Delta U \approx \rho Q_{x}$.

Из изложенного вытекает вывод о том, материнский кристалл можно рассматривать как основное состояние для МС. При этом избыточная внутренняя энергия стекла по отношению к материнскому кристаллу определяется упругой энергией системы дефектов, вмороженных при закалке расплава. Исчезновение этих дефектов при кристаллизации вызывает выделение этой энергии в виде теплоты кристаллизации.

\section{5. Релаксация модуля сдвига и тепловые эффекты ниже и выше $T_{g}$}

Рассмотрим нагрев МС от комнатной температуры в состояние переохлажденной жидкости, то есть в область температур $T_{g}<T<T_{x}$, где $T_{g}$ и $T_{x}-$ температуры стеклования и начала кристаллизации. Состояние стекла при комнатой температуре и при некоторой температуре в области переохлажденной жидкости можно охарактеризовать модулями сдвига $G_{r t}$ и $G_{s q l}$ соответственно. Пусть $\mu_{r t}$ и $\mu_{s q l}$ - модули сдвига материнского кристалла при тех же температурах. Расчет в рамках МТ дает выражение для теплоты, требуемой для нагрева от комнатной температуры в состояние переохлажденной жидкости [38]:

$$
Q=\frac{1}{\beta \rho}\left(G_{r t}-G_{s q l}+\mu_{r t}-\mu_{s q l}\right) .
$$




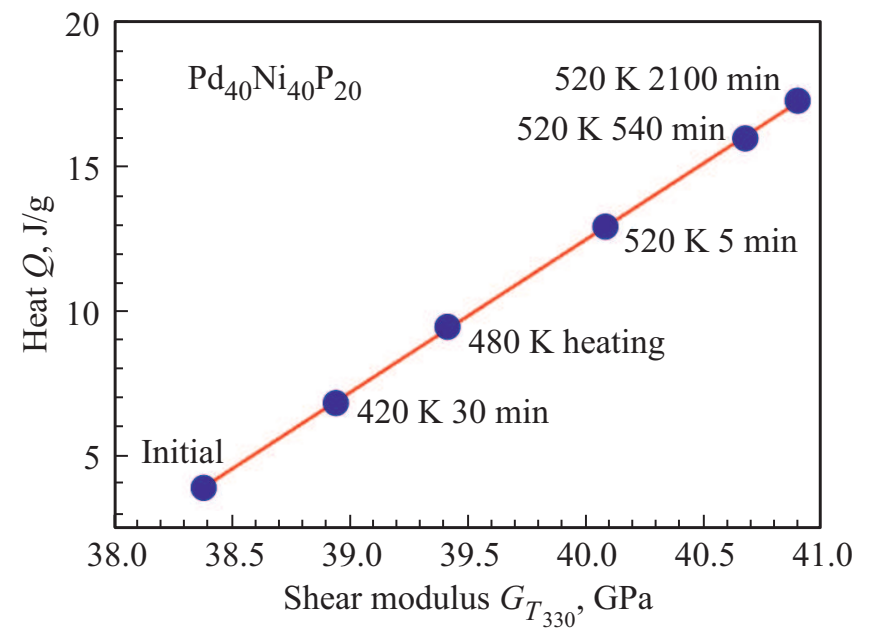

Рис. 5. Зависимость теплоты, поглощаемой при нагреве стекла $\mathrm{Pd}_{40} \mathrm{Ni}_{40} \mathrm{P}_{20}$ от 330 до $610 \mathrm{~K}$ (состояние переохлажденной жидкости) в зависимости от модуля сдвига, измеренного при $330 \mathrm{~K}$ сразу после начала нагрева. Точки соответствуют различным режимам предварительной термообработки образцов. Ошибки измерений меньше размеров символов. Прямая линия дает аппроксимацию методом наименьших квадратов [38].

Измерив модули сдвига $G_{r t}, G_{s q l}, \mu_{r t}, \mu_{s q l}$ и определив теплоту $Q$, можно проверить выполнимость соотношения (5). При этом модуль сдвига стекла при комнатной температуре $G_{r t}$ можно изменять посредством предварительной термообработки. Соответствующие эксперименты изложены в работе [38], основной результат которой показан на рис. 5. На рисунке представлена теплота, требуемая для нагрева $\mathrm{MC} \mathrm{Pd}_{40} \mathrm{Ni}_{40} \mathrm{P}_{20}$ от $T=330 \mathrm{~K}$ (эта температура более удобна для проведения экспериментов в сравнении с комнатной температурой) до температуры $T=610 \mathrm{~K}$, принадлежащей интервалу переохлажденной жидкости. Точки на этом рисунке соответствуют различным режимам предварительной термообработки образцов. Как видно, зависимость теплоты $Q$ от модуля сдвига, измеренного при $330 \mathrm{~K}$, хорошо аппроксимируется прямой линией. Возвращаясь к соотношению (5), можно отметить, что входящий в него модуль сдвига $G_{s q l}$ в состоянии переохлажденной жидкости при фиксированной температуре является константой, не зависящей от предварительной термообработки. Модули сдвига материнского кристалла $\mu_{r t}$ и $\mu_{s q l}$ при фиксированных температурах также являются константами. Соответственно, зависимость $Q\left(G_{r t}\right)$ должна быть прямой линией, что и имеет место в действительности (см. рис. 5). При этом наклон этой прямой, в соответствии с уравнением (5), должен быть равен $(\beta \rho)^{-1}$. Принимая значения плотности указанного стекла $\rho=9405 \mathrm{~kg} / \mathrm{m}^{3}$ и его сдвиговую восприимчивость $\beta=20.5$, получаем значение этого наклона равным $5.2 \cdot 10^{-6} \mathrm{~m}^{3} / \mathrm{kg}$. Это в пределах 2-3\% ошибки совпадает с экспериментальным наклоном $5.3 \cdot 10^{-6} \mathrm{~m}^{3} / \mathrm{kg}$, соответствующим представ- ленным на рис. 5 данным [38]. При этом свободный член, отвечающий линейной зависимости (5), должен быть равен $\frac{1}{\beta \rho}\left(-G_{s q l}+\mu_{r t}-\mu_{s q l}\right)$. Принимая экспериментальные значения модулей сдвига, входящих в это выражение, можно получить величину $-198 \mathrm{~kJ} / \mathrm{kg}$. то с ошибкой около $1 \%$ совпадает с данными эксперимента на рис. $5(-200 \mathrm{~kJ} / \mathrm{kg})$ [38].

Таким образом, выражение (5) дает хорошее описание эксперимента. Поскольку в это выражения входят модули сдвига $\mu_{r t}$ и $\mu_{s q l}$, мы опять приходим к выводу о том, что свойства стекла зависят от свойств материнского кристалла.

\section{6. Кинетика изменений плотности при структурной релаксации и кристаллизации}

Интерпретация изменений плотности МС при структурной релаксации и кристаллизации основана на ожидаемом изменении объема $\Delta V$ при создании дефекта межузельного типа, $\Delta V / \Omega=\alpha_{i}-1$, где $\Omega$ - объем в расчете на атом и $\alpha_{i}$ - так называемый релаксационный объем, учитывающий релаксацию структуры [39]. Тогда концентрация дефектов приведет к относительному увеличению объема на $\Delta V / V=\left(\alpha_{i}-1\right) c$. Это соотношение, с учетом связи модулей сдвига стекла и кристалла с концентрацией дефектов, даваемой формулой (1), приводит к относительному изменению плотности в виде [40]

$$
\frac{\Delta \rho}{\rho}=-\frac{\Delta V}{V}=\frac{\alpha_{i}-1}{\beta} \ln \left(\frac{\mu}{G}\right),
$$

где $\beta-$ сдвиговая восприимчивость. Зная температурные зависимости $G(T)$ и $\mu(T)$, а также оценив величину $\alpha_{i} \approx 1.4$ [40], можно рассчитать температурную зависимость относительного изменения плотности. Результат расчета для $\mathrm{MC} \mathrm{Zr}_{47} \mathrm{Cu}_{45} \mathrm{Al}_{7} \mathrm{Fe}_{1}$ показан на

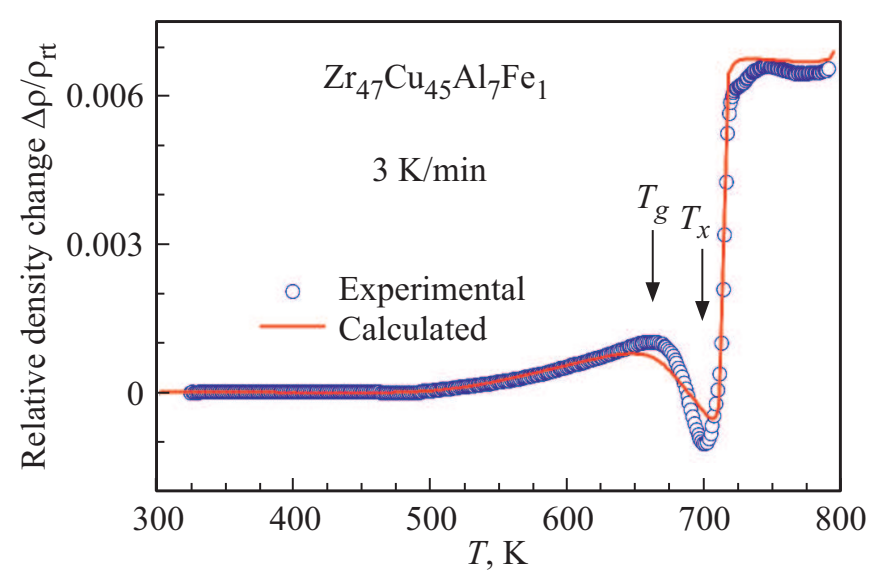

Рис. 6. Изменения плотности при нагреве $\mathrm{MC} \mathrm{Zr}_{47} \mathrm{Cu}_{45} \mathrm{Al}_{7} \mathrm{Fe}_{1}$, определенные экспериментально и рассчитанные по формуле (6) при $\alpha_{i}=1.4$ и $\beta=19.2$. Температуры стеклования и кристаллизации показаны стрелками. Данные - авторов. 
рис. 6 совместно с экспериментальными данными для того же стекла. Как видно, расчет довольно хорошо описывает все особенности релаксации - рост плотности при структурной релаксации ниже $T_{g}$, ее снижение в интервале $T_{g}<T<T_{x}$ переохлажденной жидкости и повторный рост в результате кристаллизации при $T>T_{x}$. Поскольку расчетная формула (6) включает модуль сдвига материнского кристалла, можно вновь прийти к выводу о наличии связи его свойств со свойствами приготовленного из него стекла.

\section{7. Заключение}

Выше были рассмотрены несколько наиболее убедительных примеров, свидетельствующих о наличии генетической взаимосвязи тепловых и упругих свойств металлических стекол. Главный вывод из приведенных результатов в контексте цели настоящей работы состоит в подтверждении достаточно очевидной связи упругих свойств металлических стекол и материнских кристаллов. Эта связь определяет взаимозависимость и других свойств. Материнский кристалл в энергетическом смысле можно интерпретировать как основное состояние стекла. Его избыточная внутренняя энергия определяется в основном упругой энергией системы дефектов межузельного типа, возникающих при плавлении и последующей закалке расплава. При уменьшении эффективной концентрации этих дефектов в результате структурной релаксации или кристаллизации эта энергия выделяется в виде тепла. Изменение этой концентрации приводит также к изменению плотности. Идеологической основой для понимания обсуждаемой связи „стекло-кристалл“ является межузельная теория.

\section{Список литературы}

[1] J. Schroers. Phys. Today 2, 30 (2013).

[2] W.L. Johnson. Nature Mater. 14, 553 (2015).

[3] Y.Q. Cheng, E. Ma. Prog. Mater. Sci. 56, 379 (2011).

[4] W.H. Wang. Prog. Mater. Sci. 57, 487 (2012).

[5] C. Liu, E. Pineda, D. Crespo. Metals 5, 1073 (2015).

[6] B. Ruta, E. Pineda, Z. Evenson. J. Phys.: Condens. Matter 29, 503002 (2017).

[7] A. van den Beukel, S. Radelaar. Acta Met. 31, 419 (1983).

[8] S.S. Tsao, F. Spaepen. Acta Met. 33, 881 (1985).

[9] Y.Q. Cheng, E. Ma. Appl. Phys. Lett. 93, 051910 (2008).

[10] D.B. Miracle, T. Egami, K.M. Flores, K.F. Kelton. MRS Bull. 32, 629 (2007).

[11] M.L. Falk, J.S. Langer. Phys. Rev. E 57, 7192 (1998).

[12] W. Li, Y. Gao, H. Bei. Sci. Rep. 5, 14786 (2015).

[13] D.P. Wang, Z.G. Zhu, R.J. Xue, D.W. Ding, H.Y. Bai, W.H. Wang. J. Appl. Phys. 114, 173505 (2013).

[14] A.V. Granato. Phys. Rev. Lett. 68, 974 (1992).

[15] J. Holder, A.V. Granato, L.E. Rehn. Phys. Rev. Lett. 32, 1054 (1974).

[16] J. Holder, A.V. Granato, L.E. Rehn. Phys. Rev. 10, 363 (1974).
[17] W.G. Wolfer. Fundamental Properties of Defects in Metals. In: Comprehensive Nuclear Materials / Ed. R.J.M. Konings. Elsevier: Amsterdam (2012).

[18] W. Schilling. J. Nucl. Mater. 69, 465 (1978).

[19] M. Born. J. Chem. Phys. 7, 591 (1939).

[20] E.V. Safonova, Yu.P. Mitrofanov, R.A. Konchakov, A.Yu. Vinogradov, N.P. Kobelev, V.A. Khonik. J. Phys.: Condens. Matter 28, 215401 (2016).

[21] Е.В. Гончарова, А.С. Макаров, Р.А. Кончаков, Н.П. Кобелев, В.А. Хоник. Письма в ЖЭТФ 106, 39 (2017).

[22] K. Nordlund, Y. Ashkenazy, R.S. Averback, A.V. Granato. Europhys. Lett. 71, 625 (2005).

[23] Р.А. Кончаков, Н.П. Кобелев, В.А. Хоник, А.С. Макаров. ФTT 58, 209 (2016).

[24] E.V. Goncharova, R.A. Konchakov, A.S. Makarov, N.P. Kobelev, V.A. Khonik. J. Phys.: Condens. Matter 29, 305701 (2017).

[25] A.V. Granato. Eur. J. Phys. 87, 18 (2014).

[26] S.V. Khonik, A.V. Granato, D.M. Joncich, A. Pompe, V.A. Khonik. Phys. Rev. Lett. 100, 065501 (2008).

[27] A.V. Granato. J. Non-Cryst. Solids 156, 402 (1993).

[28] P.H. Dederichs, C. Lehman, H.R. Schober, A. Scholz, R. Zeller. J. Nucl. Mater. 69, 176 (1978).

[29] A.V. Granato. J. Non-Cryst. Solids 352, 4821 (2006).

[30] C. Oligschleger, H.R. Schober. Phys. Rev. B 59, 811 (1999).

[31] H.R. Schober. J. Non-Cryst. Solids 307-310, 4049 (2002).

[32] E.V. Goncharova, R.A. Konchakov, A.S. Makarov, N.P. Kobelev, V.A. Khonik. J. Phys.: Condens. Matter 29, 305701 (2017).

[33] N.P. Kobelev, V.A. Khonik. J. Non-Cryst. Solids 427, 184 (2015).

[34] A.S. Makarov, Yu.P. Mitrofanov, G.V. Afonin, N.P. Kobelev, V.A. Khonik. Intermetallics 87, 1-5 (2017).

[35] V.A. Khonik. Chin. Phys. B 26, 016401 (2017).

[36] Y.P. Mitrofanov, D.P. Wang, A.S. Makarov, W.H. Wang, V.A. Khonik. Sci. Reports 6, 23026 (2016).

[37] G.V. Afonin, Yu.P. Mitrofanov, A.S. Makarov, N.P. Kobelev, W.H. Wang, V.A. Khonik. Acta Mater. 115, 204 (2016).

[38] A.S. Makarov, G.V. Afonin, Yu.P. Mitrofanov, R.A. Konchakov, N.P. Kobelev, J.C. Qiao, V.A. Khonik. J. Non-Cryst. Solids 500, 129 (2018).

[39] C.A. Gordon, A.V. Granato, R.O. Simmons. J. Non-Cryst. Solids 205-207, 216-220 (1996).

[40] E.V. Goncharova, R.A. Konchakov, A.S. Makarov, N.P. Kobelev, V.A. Khonik. J. Non-Cryst. Solids 471, 396 (2017).

Редактор Т.Н. Василевская 